\title{
Influence of auriculotherapy on erectile dysfunction: commentaries and perspectives
}

\author{
Matias Noll ${ }^{1,2}$, Carolina Rodrigues de Mendonça ${ }^{2}$, Priscilla Rayanne e Silva Noll ${ }^{1,3}$, \\ Erika Aparecida Silveira ${ }^{2}$
}

${ }^{1}$ Instituto Federal Goiano (IF Goiano), Ceres, Brazil; ${ }^{2}$ Departamento de Clínica Médica, Faculdade de Medicina, Universidade Federal de Goiás (UFG), Goiânia, Brazil; ${ }^{3}$ Departamento de Obstetrícia e Ginecologia, Faculdade de Medicina (FMUSP), Universidade de São Paulo, São Paulo, Brazill

Correspondence to: Matias Noll. Instituto Federal Goiano (IF Goiano), Rua 17, Qd 53, Lt 10A, Centro, Rialma, GO, Brazil.

Email: matias.noll@ifgoiano.edu.br.

Submitted Nov 03, 2019. Accepted for publication Dec 10, 2019.

doi: $10.21037 /$ tau. 2019.12 .18

View this article at: http://dx.doi.org/10.21037/tau.2019.12.18

Erectile dysfunction, defined as the inability to attain and/ or maintain penile erection sufficient for satisfactory sexual performance (1), is the most common sexual problem discussed in the literature (2) and affects about half of adult men (3). Treating sexual dysfunctions, especially erectile dysfunction, with alternative and complementary therapies seem desirable in clinical practice, and traditional Chinese medical therapy has been applied with success for erectile dysfunction (4). Engelhardt et al. (5) investigated the effect of acupuncture in patients with erectile dysfunction and found benefits in more than two-thirds of those with erectile dysfunction. In contrast to alternative medicine, the use of medications for erectile dysfunction increases feelings of being "less of a man" in that the patient is considers that it is the pill, and not him that achieves the erection.

Given that auriculotherapy is a safe, simple, and lowcost technique used for a wide variety of conditions (6), it may also be a treatment option for sexual dysfunctions. Auriculotherapy is a treatment modality involving complementary and alternative medicine based on the stimulation of reflex points in the ear. Further, auriculotherapy, also known as auricular acupuncture, is based on the stimulation of ear points for therapeutic purposes using acupuncture. This modality is used to treat several conditions, including chronic and acute pain, temporomandibular dysfunction, anxiety, and insomnia $(7,8)$. Recent studies have suggested that auriculotherapy has some beneficial effects on weight reduction (9). Considering the high prevalence of erectile dysfunction, it is important to review previous epidemiological and clinical trials to analyze the effectiveness of auriculotherapy for erectile dysfunction. We aimed to clarify if auriculotherapy could reduce erectile dysfunction and improve the psychosocial wellbeing of patients in relation to erectile dysfunction.

Consequently, we performed a systematic review to analyze the influence of auriculotherapy on erectile dysfunction. We considered eligible studies that investigated the effects of auriculotherapy on erectile dysfunction. The eligibility criteria were specified according to the PICO (Population, Intervention, Comparison, Outcome) structure, which is explained in the recommended notification items for systematic reviews. Accordingly, "P" represented adult, "I" represented the use of auriculotherapy, "C" represented the use of other therapies or placebo, and "O" represented erectile dysfunction variables. Observational studies and clinical trials were included with no restrictions regarding language or year of publication. The exclusion criteria were as follows: studies with incomplete data and review articles.

We performed three databases (SCOPUS, PubMed, and Science Direct) searches, and the following combination of keywords were used in PubMed: "(((auriculotherapy[MeSH Terms]) OR "auricular acupuncture"[Title/Abstract])) AND ((((("Sexual Dysfunctions" [Title/Abstract]) OR "male Impotence" [Title/Abstract]) OR "Male Impotence "[Title/ Abstract]) OR "Male Sexual Impotence"[Title/Abstract]) OR erectile dysfunction[MeSH Terms])"; Scopus: "TITLEABS-KEY(auriculotherapy OR "auricular acupuncture") 
AND TITLE-ABS-KEY("Sexual Dysfunctions" OR "male Impotence" OR "Male Impotence" OR "Male Sexual Impotence" OR "erectile dysfunction")"; and Science Direct: "(auriculotherapy OR "auricular acupuncture") AND ("Sexual Dysfunctions" OR "male Impotence" OR Male Impotence" OR "Male Sexual Impotence" OR "erectile dysfunction")".

Six eligible studies were identified (PubMed, 1; Scopus, 5; Science Direct, 0). Subsequently, we analyzed both the titles and abstracts of the identified studies to decide which would be included in our systematic review. After reading the full articles, only one article (10) met our criteria. As only one study was found, performing a systematic review was infeasible; however, we considered it relevant to share our findings in this letter to the editor.

Suenson (10), in a case study, applied a 20 -week multidisciplinary approach using auriculotherapy in 19 participants, which also included practical home exercises. The study findings suggest that the program helped to improve both erectile function and self-esteem. Moreover, the social relationships of the patients were also improved as demonstrated via the self-assessment data. In conclusion, although this study showed the benefits of auriculotherapy, these data are only initial indicators as studies are lacking in this field and more research is required to provide enough evidence about this alternative treatment for this prevalent health problem.

Understanding the effects of auriculotherapy on sexual dysfunctions is important because it should help health professionals in the clinical setting. Further scientific inquiry into the mechanisms of action and clinical applications of auriculotherapy seems necessary. In addition, it is important to expand on both the professional and public education of the merits of these techniques for erectile dysfunction.

\section{Acknowledgments}

The authors thank to the Instituto Federal Goiano [www. ifgoiano.edu.br] and the Child and Adolescent Health Research Group (GPSaCA) - www.gpsaca.com.br for the support.

\section{Footnote}

Conflicts of Interest: The authors have no conflicts of interest to declare.

Ethical Statement: The authors are accountable for all aspects of the work in ensuring that questions related to the accuracy or integrity of any part of the work are appropriately investigated and resolved.

\section{References}

1. NIH Consensus Conference. Impotence. NIH Consensus Development Panel on Impotence. JAMA 1993;270:83-90.

2. Huang YP, Chen B, Ping P, et al. Asexuality development among middle aged and older men. PLoS One 2014;9:e92794.

3. Greenberg DR, Richardson MT, Tijerina JD, et al. The Quality of Systematic Reviews and Meta-Analyses in Erectile Dysfunction Treatment and Management. J Sex Med 2019;16:394-401.

4. Li H, Jiang H, Liu J. Traditional Chinese medical therapy for erectile dysfunction. Transl Androl Urol 2017;6:192-8.

5. Engelhardt PF, Daha LK, Zils T, et al. Acupuncture in the treatment of psychogenic erectile dysfunction: first results of a prospective randomized placebo-controlled study. Int J Impot Res 2003;15:343-6.

6. Franco FRV, Castro LA, Borsatto MC, et al. Combined Acupuncture and Auriculotherapy in Burning Mouth Syndrome Treatment: A Preliminary Single-Arm Clinical Trial. J Altern Complement Med 2017;23:126-34.

7. Dellovo AG, Souza LMA, de Oliveira JS, et al. Effects of auriculotherapy and midazolam for anxiety control in patients submitted to third molar extraction. Int J Oral Maxillofac Surg 2019;48:669-74.

8. Franco FR, Castro LA, Borsatto MC,et al. Combined Acupuncture and Auriculotherapy in Burning Mouth Syndrome Treatment: A Preliminary Single-Arm Clinical Trial. J Altern Complement Med 2017;23:126-34.

9. Mendonça CR, Coelho Dos Santos LS, Noll M, et al. Effects of auriculotherapy on weight and body mass index reduction in patients with overweight or obesity: Systematic review and meta-analysis. Complement Ther Clin Pract 2019. [Epub ahead of print].

10. Suenson S. A Multidisciplinary Approach Using Auriculotherapy for Erectile Dysfunction: A Qualitative Case Study. Med Acupunct 2014;26:189-96.

Cite this article as: Noll M, de Mendonça CR, Noll PRES, Silveira EA. Influence of auriculotherapy on erectile dysfunction: commentaries and perspectives. Transl Androl Urol 2019;8(Suppl 5):S550-S551. doi: 10.21037/tau.2019.12.18 